\title{
PATRONES POBLACIONALES DE LAS PRINCIPALES ESPECIES HERBÁCEAS EN LA RESERVA NACIONAL DE LACHAY
}

\section{POPULATION PATTERNS OF THE MAIN HERBACEOUS SPECIES AT LACHAY NATIONAL RESERVE}

\author{
Vanessa Teixeira Roth ${ }^{1}$ y Edgar Sánchez Infantas ${ }^{2}$
}

\begin{abstract}
Resumen
La Ley de potencia de Taylor tiene como base la relación entre la densidad media de una población y su variabilidad espacial y temporal, en otras palabras es una relación empírica entre la varianza y la media de estimaciones de abundancia, descrita mediante la ecuación potencial $\mathrm{S}^{2}=\mathrm{a}$ $\mathrm{mb}$, donde $\mathrm{S}^{2}$ y $\mathrm{m}$ representan la varianza y la media, respectivamente, mientras que a y b son parámetros a estimar estadísticamente. La Ley de Taylor permite cuantificar el grado de agregación mediante la pendiente del modelo indicando si las poblaciones cumplen con patrones uniforme, aleatorio y agregado. Redfern \& Pimm (1988) reemplazaron la varianza por el coeficiente de variabilidad y McArdle (1990) lo utilizó para generar una nueva ecuación de la ley de Taylor. El presente estudio se realizó en la Reserva Nacional de Lachay entre Febrero de 1998 y Julio de 1999 durante el cual se realizó un monitoreo constante de las variaciones espaciotemporales de los patrones poblacionales de la comunidad herbácea de este ecosistema. Basándonos en la Ley de Taylor se determinaron los principales patrones poblacionales de las especies determinantes de la comunidad herbácea de las lomas de Lachay, así como su variación espacio-temporal ante eventos perturbadores como El Niño (1997-1998). Como resultado se determinó el patrón poblacional de 16 especies vegetales de la comunidad florística de Lachay, encontrándose 5 especies con patrón "hotspots moviles o fijos", 3 especies con patrón "refugios" y 3 especies con patrón "hotspots". Estos patrones están influenciados por las condiciones meteorológicas y físicas del ambiente, como la humedad, precipitación y radiación solar.

Palabras claves: Evento El Niño, variación espacio-temporal, Ley de Taylor y "Hotspots"
\end{abstract}

\begin{abstract}
Taylor's power has its bases in the relationship between the average density of a population and its space-temporary variation. In other words it is an empirical relationship between the variance and average of abundance estimations. This is described by the potential ecuation $\mathrm{S}^{2}=\mathrm{a}$ $\mathrm{mb}$, where $\mathrm{S}^{2}$ and $\mathrm{m}$ represent the variance and the average, respectively, whereas $\mathrm{a}$ and $\mathrm{b}$ are parameters to be estimated statistically. The Law of Taylor allows to quantify the degree of aggregation by means of the slope of the model indicating if population patterns are uniform, random or aggregate. Readfern and Pimm (1988) replaced the variance by the variability coefficient, and McArdle (1990) used it to generate a new equation of the Taylor's Law. The present study was conducted at Lachay National Reserve from February 1998 to July 1999 during which a constant evaluation of the space-temporary variations of the population patterns of the herbaceous community of this ecosystem was made. With this research we determined the main population patterns of the determining species of the herbaceous community of Lachay, as well as their space-temporary variations in a disturbing event such as "El Niño" (1997-1998). As a result we appreciated the population pattern of 16 plant species of the herbaceous community of Lachay: 5 species had a "fixed or moving hotspots" pattern, 5 species had a "refuge" pattern and 3 species had a "hotspots" pattern. These patterns are influenced by meteorological and physical conditions of the atmosphere, such as moisture, precipitation and solar radiation.
\end{abstract}

Key words: "El Niño" event, space-temporary variation, Taylor Power Lay and hotsposts

\section{Introducción}

Taylor documentó la existencia de una relación positiva entre la media y la varianza en las medidas de densidad de varias poblaciones naturales (BenedettiCecchi, 2003). La Ley de potencia de Taylor trata de predecir el grado de correlación entre los individuos y la pendiente de la varianza, por lo tanto refleja el grado de correlación entre individuos de una población (Kerkhoff \& Ballantyne, 2003).

Como se mencionó anteriormente la ley de Taylor es una ley empírica basada en la relación entre la varianza muestral espacial o temporal $\left(\mathrm{S}^{2}\right)$ y la media muestral (m) de las densidades muestrales y fue propuesta por Taylor (1961). Después debido a 
diferentes estudios como el de Redfern \& Pimm (1988) propusieron reemplazar a la varianza por el coeficiente de variabilidad, como medida de variabilidad poblacional, ya que este no se ve afectado por los ceros y admite la comparación entre especies (Giraldo et al., 2002). Esta ley permite entender los patrones de distribución espacio-temporal de las poblaciones sugiriendo además los procesos que pueden generar estos patrones. Para describir la variabilidad poblacionales espacial o temporal se utiliza el coeficiente de variabilidad (CV); la comparación de la variabilidad de una población con su densidad media permite identificar procesos como la existencia de refugios en la población o "hotspots" (puntos de crecimiento poblacional súbito) en alguna parte del área de distribución de las poblaciones en estudio (McArdle et al., 1990).

Mc Ardle et al. (1990) utilizaron esta variación de la ley de Taylor para describir variaciones espaciales o temporales, donde se tiene un $\beta$ espacial $(\beta s)$ cuando se mide la varianza en parcelas determinadas en diferentes tiempos y un $\beta$ temporal $(\beta \mathrm{t})$ que es la variación de las especies en el tiempo.

Para determinar los patrones poblacionales con la Ley de Taylor, como indica Mc Ardle et al. (1990) basándose en interpretaciones previas de Soberón \& Loevinsohn (1987) se tiene que tomar en cuenta que:

- Si $\beta \mathrm{s}<2, \beta \mathrm{t}>2$ : La población en las parcelas de mejor calidad es más variables en el tiempo que en las parcelas de baja calidad. Pero en años buenos hay menor variabilidad. Este tipo de patrón necesita un mayor análisis empírico y teórico, por lo tanto todavía no se entiende bien.

- $\quad$ Si $\beta s>2, \beta t<2$ : La población en parcelas de buena calidad es menos variable en el tiempo. En años estables hay más variabilidad en el espacio. Las parcelas de buena calidad actúan como "hotspost móviles o fijos".

- $\quad$ Si $\beta s>2, \beta t>2$ : La población en las parcelas de mejor calidad es más variable que en las de baja calidad. En años estables hay más variabilidad. Las parcelas de buena calidad se incrementan y actúan como "hotspost".

- $\quad$ Si $\beta \mathbf{s}<2, \beta \mathrm{t}<2$ : La población en las parcelas de buena calidad es menos variables en el tiempo, y en los años menos estables hay mayor variabilidad espacial, actuando en forma de "refugio"; en los años inestables los sitios de mejor calidad se mantienen.

Por lo tanto, para caracterizar el patrón espacial de una población mediante la ley de Taylor, es necesario contar con un conjunto de muestreos, cada uno de los cuales haya arrojado una estimación de la media y la varianza de la densidad poblacional.

En estudios de población, la identificación de patrones poblacionales ayuda a descubrir mecanismos biológicos que contribuyen al ordenamiento espacial de los individuos (Matteuci, 1982). La variabilidad de cada población se basa en diferentes componentes que afectan a la especie en el espacio y en el tiempo, además las poblaciones biológicas tienen un amplio margen de comportamiento. Es por eso que la habilidad para comparar poblaciones con parámetros ecológicos sirve para entender por qué diferentes especies pueden tener dinámicas poblacionales similares (McArdle et al., 1990).

Las poblaciones vegetales de las cuales se obtuvieron los datos se encuentran en la Reserva Nacional de Lachay a $105 \mathrm{~km}$ al norte de Lima, entre las ciudades de Chancay y Huacho. La formación vegetal denominada "lomas" se caracteriza por ser de porte bajo, conformadas por vegetación herbácea, arbustiva y arbórea, de expansión limitada y fuerte periodicidad, ubicadas en forma dispersa a lo largo de la Costa (Torres, 1984). Estos ecosistemas se ven influenciados por perturbaciones naturales como el evento "El Niño" que es un fenómeno complejo, meteorológico, oceanográfico y cíclico que se inicia a fin de año cuando ocurre un debilitamiento anómalo de los vientos alisios ligados al anticiclón superficial del hemisferio sur y tiene manifestaciones patentes en el Perú (Vegas, 1996).

Una perturbación es todo aquello que afecta la organización de un ecosistema, por lo tanto el evento "El Niño" como cualquier otra perturbación natural o artificial desorganiza la estructura y funcionalidad del sistema. Sin embargo, estos cambios configuran el futuro desarrollo del ecosistema (Walter, 2000).

El objetivo de este estudio es determinar los patrones poblaciones de las principales especies herbáceas, basándonos en la Ley de Potencia de Taylor, tomando en cuenta la repercusión del evento "El Niño" (1997-1998) y la variación de estos patrones en el espacio y el tiempo.

\section{Materiales y Métodos}

\section{Área de Estudio}

La Reserva Nacional de Lachay se encuentra ubicada entre los $11^{\circ} 21^{\prime}-11^{\circ} 21^{\prime} 58^{\prime}$ ' L.S y $77^{\circ} 21^{\prime} 28^{\prime}$ ', - 77²2’25' L.O (Ferreira, 1985); y comprende los distritos de Sayán, Chancay, Huacho y Huaral de la Provincias de Chancay y Huaral. Para determinar las variaciones espacio-temporales de las poblaciones en la R.N. de Lachay se utilizó el muestreo aleatorio sistemático (Ramírez, 1999). Primero se dividió el área de estudio en tres grandes zonas de tamaño uniforme, tomando de base tanto la forma del relieve como la cantidad de visitantes que las frecuentaban. Dentro de estas tres zonas se ubicaron 31 unidades muestrales de $1 \mathrm{~m}$ cuadrado, repartidas en 12,11 y 8 unidades muéstrales en cada una de las tres zonas. En cada parcela se tomaron datos de cobertura, número de especies, altura y características físicas como pendiente y exposición. Se utilizó un marco de madera de $1 \mathrm{~m}^{2}$ para delimitar la unidad muestral; el tamaño de la unidad muestral se determinó en base a curvas de 
especies-áreas. Cada registro demoró 3 días y se realizaron en los meses de Febrero, Mayo, Agosto y Diciembre de 1998 y Febrero y Julio de 1999. Se eligieron estos meses ya que representan las variaciones estaciones a lo largo del año, observando el efecto del evento de El Niño durante 1998. Se obtuvieron datos meteorológicos mensuales de temperatura media mensual, humedad relativa media mensual y precipitación mensual para los años 1997, 1998 y 1999 de la estación meteorológica de la R.N. de Lachay.

Índice de Valor de Importancia

El índice de valor de importancia (IVI), se utiliza para el análisis de los parámetros ecológicos ya que es un buen descriptor de la importancia de la especie en el lugar. El IVI es un parámetro adimensional (Matteuci, 1982).

La formula que se utilizó fue:

IVI $=$ Densidad $(\mathrm{d})+$ Cobertura $(\mathrm{cm} 2)$

Donde: $\mathrm{d}=$ individuos/metro cuadrado

Patrón Poblacional

Con una matriz de doble entrada de parcelas versus tiempo se encontró la densidad media $(\mathrm{N})$ y el coeficiente de variabilidad (CV) espacial y temporal de las especies más importantes. Teniendo estos datos se gráficó el $\log (\mathrm{N})$ vs $\log (\mathrm{CV})$ y por medio de una regresión lineal se obtiene los valores de $\beta$, que es un parámetro utilizado para medir la variabilidad de la población en función de la densidad media $(\mathrm{N})$. Cuando $\beta$ es igual a 2 la variabilidad de la población no depende de N (Mc Ardle et al., 1990).

$$
\begin{aligned}
& \mathrm{CV}=\alpha^{0.5} \mathrm{~N}^{(0.5 \beta-1)}, \text { linealizando } \\
& \log \mathrm{CV}=0.5 \log \alpha+(0.5 \beta-1) \log \mathrm{N} \\
& \mathrm{b}=0.5 \beta-1 \text { donde: } \mathrm{b} \text { : pendiente }
\end{aligned}
$$

$\underline{\text { Resultados y discusión }}$

En la Tabla 1, se observan los valores de IVI totales para las 16 principales especies herbáceas de Lachay; aquí se indica cuales fueron las especies dominantes durante el periodo de investigación. En la Tabla 2 se observa la aplicación de la Ley de Taylor en las poblaciones de plantas de la Reserva Nacional de Lachay. Del análisis efectuado se puede concluir que de las 16 especies herbáceas más importantes en Lachay, cinco de ellas, Solanum phyllantum, Urocarpidium peruvianum, Lycopersicon peruvianum, Poa sp. y Nicotiana paniculata actúan como "hotspots moviles o fijos", donde $\beta \mathrm{s}>2$ y $\beta \mathrm{t}<2$; esto indica que durante los periodos ambientales estables, cuando la densidad media (N) aumenta, algunas parcelas registran un crecimiento poblacional y esto incrementa la variabilidad espacial. La mayoría de la especies "hotspots móviles o fijos" como $N$. paniculata y $U$. peruvianum tienen un alto índice de valor deiImportancia (IVI) totales. Estas especies se caracterizan por ser menos variables antes perturbaciones naturales como el evento "El Niño", comportándose como núcleos de densidad dinámicos cumpliendo una función esencial en el mantenimiento de las poblaciones de las especies. Especies como Vasquezia oppositifolia, Sicyos baderoa, Lycopersicon peruvianus y Loasa urens actúan dentro del patrón "hotspost", esto quiere decir que debido a las buenas condiciones climáticas que trajo el evento "El Niño" estas especies tuvieron un crecimiento explosivo, ubicándose espacialmente en todo el área y temporalmente tuvieron un gran crecimiento estacional, pero luego decrecieron. Algunas de estas especies se mantuvieron fijas espacial y temporalmente como Lycopersicon peruvianus y Loasa ureas, demostrando valores relativamente altos de IVI, sin embargo las otras especies como Vasquezia oppositifolia y Sicyos baderoa desaparecen virtualmente después de su "hotspost" y lo que es demostrado por sus pequeños valores totales de IVI. El patrón de estallido $u$ explosión, $\beta \mathrm{s}>2$ y $\beta \mathrm{t}>2$, es el que comúnmente tienen las plantas plagas, las malezas $\mathrm{y}$ en los sitios no perturbados las poblaciones aumentan. Estas especies se caracterizan por su marcada estacionalidad.

Las especies "hotspots móviles o fijos" y "hotspots" se reemplazan en el tiempo, como se puede apreciar en la Figura 1a, originando una dinámica sucesional interesante de investigar, ya que las especies con patrones "hotspots" pueden ser móviles o fijos, regulando de esta forma las densidades medias de las especies.

Tabla 1. Valores de IVI totales de las principales especies herbáceas de la Reserva Nacional de Lachay en orden descendente.

\begin{tabular}{lr}
\hline Especies Vegetales & \multicolumn{1}{c}{ IVI totales } \\
\hline Nicotiana Paniculata & 30326.929 \\
Chenopodium petiolare & 14069.2 \\
Nolana humifusa & 9457.8381 \\
Urocarpidium peruvianum & 7196.64 \\
Loasa urens & 6568.5913 \\
Hyptis sidifolia & 3771.82 \\
Lycopersicon peruvianum & 3588.79 \\
Alternanthera halimifolia & 3175.2706 \\
Croton ruizianus & 2950.8713 \\
Trixis cacalioides & 2663.8374 \\
Ophryosporus peruvianus & 2562.0452 \\
Vasquezia oppositifolia & 1423.84 \\
Poaceae sp & 1270.3671 \\
Solanum phyllantum & 592.22 \\
Sicyos baderoa & 272.08 \\
Stenomesson coccineum & 196.18032
\end{tabular}


Tabla 2. Valores de B espacial (Bs) y temporal (Bt) de la Ley de Taylor para las especies representativas de la Reserva Nacional de Lachay.

\begin{tabular}{|c|c|c|c|}
\hline Especies & Bs & Bt & Patrón \\
\hline Nicotiana paniculata & 2.0322 & 1.8118 & $\begin{array}{l}\text { Hotspost } \\
\text { móviles o } \\
\text { fijos }\end{array}$ \\
\hline Chenopodium petiolare & 1.8262 & 2.007 & $* * *$ \\
\hline Nolana humifusa & 1.9952 & 2.0516 & $* * *$ \\
\hline Urocarpidium peruvianum & 2.1988 & 1.992 & $\begin{array}{l}\text { Hotspost } \\
\text { móviles o } \\
\text { fijos }\end{array}$ \\
\hline Loasa urens & 2.2004 & 2.0298 & Hotspost \\
\hline Hyptis sidaefolia & 1.4578 & 2.0502 & $* * *$ \\
\hline Lycopersicon peruvianum & 2.1418 & 1.927 & $\begin{array}{l}\text { Hotspost } \\
\text { móviles o } \\
\text { fijos }\end{array}$ \\
\hline Althernanthera halimifolia & 1.8654 & 1.8006 & Refugio \\
\hline Croton ruizianus & 0.9726 & 2.0998 & $* * *$ \\
\hline Trixis cacalioides & 1.6456 & 2.0936 & $* * *$ \\
\hline Ophryosporus peruvianus & 1.6112 & 1.986 & Refugio \\
\hline Vasquezia oppositifolia & 2.1454 & 2.078 & Hotspost \\
\hline Poaceae sp & 2.1322 & 1.9999 & $\begin{array}{l}\text { Hotspost } \\
\text { móviles o } \\
\text { fijos }\end{array}$ \\
\hline Solanum phyllantum & 2.1328 & 1.9594 & $\begin{array}{l}\text { Hotspost } \\
\text { móviles o } \\
\text { fijos }\end{array}$ \\
\hline Sicyos baderoa & 2.0412 & 2.1068 & Hotspost \\
\hline Stenomesson coccineum & 1.9618 & 1.9356 & refugio \\
\hline
\end{tabular}

También se observa que Chenopodium petiolare, Croton ruizianus, Nolana humifusa, Trixis cacaloides e Hyptis sidaefolia tienen un patrón poblacional difícil de entender biológicamente el cual necesita de un mayor acercamiento de la ley empírica con la realidad ecológica. La variabilidad espacial de estas especies aumenta con una perturbación, sin embargo su densidad aumenta, al contrario de disminuir. Estas especies tienen un $\beta$ que no es significativamente diferente a 2 por lo que no están muy influenciadas por su densidad. También se distribuyen de manera irregular en la Tabla 1, teniendo valores de IVI muy altos, así como medio bajos., es decir no se encuentran espacialmente distribuidas en toda el área de Lachay. Sin embargo este grupo de especies cumplen también con el proceso de sucesión, como se aprecia en la Figurala.

Cuando las especies en un año perturbado tienen gran variabilidad espacial, pero se mantienen estables con poca variabilidad temporal, como Stenomesson coccineum, Althernanthera halinmifolia y Ophyosporus peruvianus, se dice que tienen un patrón de refugio, $\beta \mathrm{s}<2$ y $\beta \mathrm{t}<2$; estas especies se encuentran normalmente en las zonas bajas de Lachay; además se caracterizan por tener un valor de IVI bajo-medio pero constante en el tiempo (Tabla 1 y Figura 1b).
Los patrones poblaciones de una especie están determinados tanto por variables bióticas (propias de cada especie) como ambientales, en este caso el evento de "El Niño" (Ramírez, 1999). Los patrones poblaciones que acabamos de ver nos sirven para entender la variabilidad espacio-temporal de la comunidad vegetal, así como las distintas respuestas ambientales que tienen las diferentes especies ante una perturbación natural cíclica que tiene un impacto en el ecosistema. El proceso de sucesión que ocurre especialmente entre especies del mismo patrón, es una respuesta clara ante esta perturbación de la cual las especies ya tienen una "memoria evolutiva" y de la que escapan fácilmente. Esta sucesión que se observa claramente, es el resultado de las repuestas de las especies a esta inestabilidad ambiental puntual, como "El Niño". Esta sucesión ocupa ambientes nuevos, desprovistos temporalmente de vida, que por los procesos de organización interna y debido a una perturbación como El Niño, se logra suceder de forma fenomenológica.

Las perturbaciones eliminan o modifican algunos de los componentes del ecosistema, en este caso las poblaciones vegetales herbáceas. Cada perturbación va a actuar de diferentes maneras sobre cada una de estas especies. Sin embargo, el ecosistema que emerge de la perturbación va a tratar en algunos casos de replicar la organización previa a la perturbación y en otros va a conformar toda una nueva organización (Walter, 2000). En este caso como se observa en la Fig. 1 las especies, especialmente las que comparten un mismo patrón, adaptan comportamientos separados para poder lidiar con el evento "El Niño" y poder sobrevivir. Cada una de ellas se reorganiza y regula para entrar dentro de la dinámica temporal de la perturbación. El análisis de la Ley de Taylor sugiere que las lomas, ante la presencia del evento El Niño, actúan incorporando esta variabilidad como normal, es decir pareciera que debido a la presencia constante del Niño en la historia evolutiva de las lomas, estas han logrado retener información importante para hacer frente a esta perturbación y las poblaciones vegetales tienen patrones de respuesta que les permite responder reorganizando su espacio a la manera más adecuada posible según la información y variedad de respuesta que posean (Margalef, 1991).

\section{Conclusiones}

- Las asociaciones entre las diferentes especies herbáceas de la Reserva Nacional de Lachay, tienen en la mayoría una estacionalidad muy marcada, siendo muchas de ellas inestables en el tiempo.

- Las Lomas de Lachay, sufren un marcado proceso sucesional que se expresa fundamentalmente en el cambio de abundancias de las especies presentes, sin embargo debido a la presencia de "El Niño" no se registra un cambio estacional significativo 
de la composición florística, mas si un cambio en su distribución espacial.

- Existen diferentes patrones poblacionales: 5 especies "hotspots móviles o fijos" y 3 "refugio", que son variables en el espacio y en tiempo respectivamente, además de 5 especies, las cuales sus poblaciones no están muy influenciadas por la densidad y 3 especies con patrón "hotspost", que explican la dinámica poblacional de la comunidad y los procesos internos que sufre la comunidad herbácea de Lachay ante una perturbación como el evento de "El Niño".

- Las especies herbáceas de las lomas de Lachay, responden eficientemente ante el evento de "El Niño", respondiendo a la variabilidad de su entorno a través de variaciones estacionales o espaciales y de dinámicas de crecimiento interno a las que cada una de ellas se somete para lograr la funcionabilidad del ecosistema.

\section{Agradecimientos}

A la oficina de Investigación de la UNALM, por el apoyo financiero para la realización de las salidas de campo. Al personal de la Reserva Nacional de Lachay por las facilidades brindadas en cada uno de los muestreos.

\section{Literatura citada}

Benedetti-Cecchi L. 2003. The importance of the variance around the mean effect size of ecological processes. Ecology. 84:2335-2346.

Ferreyra R. 1985. Las Lomas de Lachay. Saint Paulis. Volumen 4. Agosto 1985. Pag 7-9. Lima-Perú.

Giraldo A., Véliz C.; Arellano G. \& Sánchez E. 2002. El uso de la ley de Taylor en el establecimiento de patrones de varación espacio-temporal en poblaciones animales: dos ejemplos de aplicación. Ecología Aplicada. 1(1): 71-74.

Kerkhoff A.J. \& Ballantyne F. 2003. The scaling of reproductive variability in trees. Ecology Letters. 6: 850856.

Margalef R. 1991. Teoría de los sistemas ecologicos. Universitat de Barcelona. España.

Matteuci S. 1982. Metodología para el estudio de la vegetación. Universidad. Nacional. Francisco de Miranda. Estado de Falcon, Caracas.

McArdle B.H., Gaston K. \& Lawton J. 1990. Variation in the size of animal population: Patterns, problems and artefacts. Journal of Animal Ecology. 59: $439-454$

Ramírez A. 1999. Ecología Aplicada. Fundación Universidad de Bogota Jorge Tadeo Lozano. Bogota- Colombia.

Redfern A. \& Pimm S. 1988. Population variability and polyphagy in herbivorous

Figura 1a. Evolución Temporal de las principales especies herbáceas con mayor IVI en la Reserva Nacional de Lachay (De Febrero 1998 a Julio 1999) (primeras 8 especies con mayor IVI).

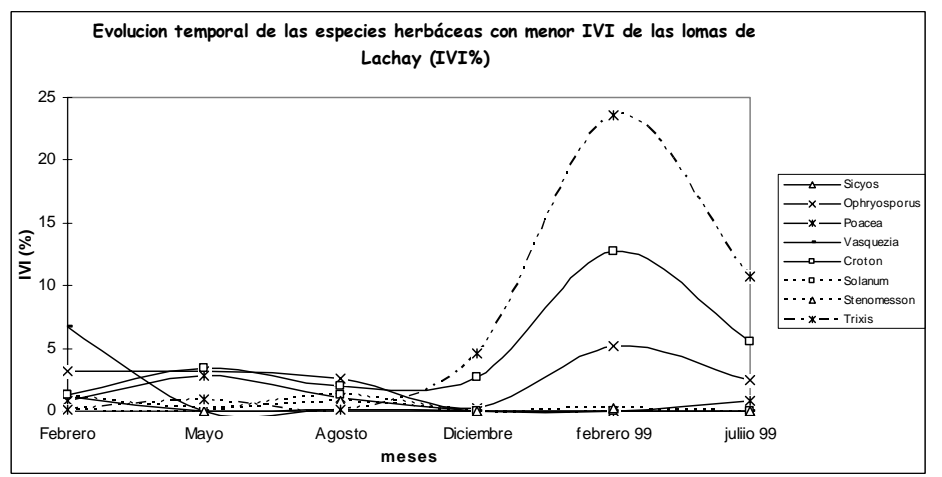

insect communities. Ecological Monographs. 58(1): 3955.

Soberón J. \& Loevinsohn M. 1987. Patterns of variation in the numbers of animal populations and the biological foundations of Taylor's law of the mean. Okos. 48: 249252.

Taylor L.R. 1961. Aggregation, variante and the mean. Nature. 189: 732-735.

Torres J. 1984. Anomalías observadas en la vegetación y sus factores físicos determinantes en las lomas de la costa central, durante el verano (Enero-Abril) de 1983.

Vegas M. 1996. Ecología y Mar Peruano. CONCYTEC. Perú.

Walter B. 2000. Analysing Integrated Social- Ecological Systems. Wallenberg Workshop. Sotckholm.

Figura 1b. Evolución Temporal de las especies herbáceas con menor IVI en la Reserva Nacional de Lachay (De Febrero 1998 a Julio 1999) (8 especies).

${ }^{1}$ Pontificia Universidad Catolica del Perú. Av. Universitaria s/n. Lima. Apartado 1761. Lima 100. E-mail: vteixeira@pucp.edu.pe

${ }^{2}$ Laboratorio de Ecología de Procesos, Dpto. de Biología. Universidad Nacional Agraría La Molina Lima. Email: esi@lamolina.edu.pe 